\title{
Blood Flow Changes Coincide with Cellular Rearrangements during Blood Vessel Pruning in Zebrafish Embryos
}

\author{
Eva Kochhan ${ }^{1}$, Anna Lenard ${ }^{2}$, Elin Ellertsdottir ${ }^{2}$, Lukas Herwig ${ }^{2}$, Markus Affolter ${ }^{2}$, Heinz-Georg Belting ${ }^{2}$, \\ Arndt F. Siekmann ${ }^{1 *}$
}

1 Max Planck Institute for Molecular Biomedicine, Laboratory for Cardiovascular Patterning, Muenster, Germany, 2 Biozentrum der Universität Basel, Abteilung Zellbiologie, Basel, Switzerland

\begin{abstract}
After the initial formation of a highly branched vascular plexus, blood vessel pruning generates a hierarchically structured network with improved flow characteristics. We report here on the cellular events that occur during the pruning of a defined blood vessel in the eye of developing zebrafish embryos. Time-lapse imaging reveals that the connection of a new blood vessel sprout with a previously perfused multicellular endothelial tube leads to the formation of a branched, $\mathrm{Y}$ shaped structure. Subsequently, endothelial cells in parts of the previously perfused branch rearrange from a multicellular into a unicellular tube, followed by blood vessel detachment. This process is accompanied by endothelial cell death. Finally, we show that differences in blood flow between neighboring vessels are important for the completion of the pruning process. Our data suggest that flow induced changes in tubular architecture ensure proper blood vessel pruning.
\end{abstract}

Citation: Kochhan E, Lenard A, Ellertsdottir E, Herwig L, Affolter M, et al. (2013) Blood Flow Changes Coincide with Cellular Rearrangements during Blood Vessel Pruning in Zebrafish Embryos. PLoS ONE 8(10): e75060. doi:10.1371/journal.pone.0075060

Editor: Ben Hogan, University of Queensland, Australia

Received July 5, 2013; Accepted August 8, 2013; Published October 11, 2013

Copyright: (c) 2013 Kochhan et al. This is an open-access article distributed under the terms of the Creative Commons Attribution License, which permits unrestricted use, distribution, and reproduction in any medium, provided the original author and source are credited.

Funding: This work was funded by the Max Planck Society (www.mpg.de; AFS), the Deutsche Forschungsgemeinschaft (DFG SI-1374/3-1; www.dfg.de; AFS) and an European Research Council starting grant (260794-ZebrafishAngio; erc.europa.eu; AFS). The funders had no role in study design, data collection and analysis, decision to publish, or preparation of the manuscript.

Competing Interests: The authors have declared that no competing interests exist.

*E-mail: arndt.siekmann@mpi-muenster.mpg.de

\section{Introduction}

The vasculature is the first organ system to form during embryonic development and meets the challenge to grow and refine while it is already functioning. After the initial sprouting of blood vessels, remodeling ensures the formation of a more efficient vascular network [1-3]. While many of the key factors and genetic players regulating blood vessel sprouting are known, the mechanisms which control blood vessel remodeling and pruning are only poorly understood. The best-studied context is the regression of the hyaloid vasculature in mice [4]. Here, macrophages secrete a WNT ligand that induces apoptosis in endothelial cells, ultimately leading to the complete removal of this vascular structure $[5,6]$. However, in many other contexts where vascular pruning has been described, such as the mouse retina [7,8], the branchial arches [9] or the zebrafish brain [10], only a subset of blood vessel connections is removed. This raises the question: Which mechanism selects the blood vessels to be pruned? In addition, it is unclear how, once a given blood vessel is selected to disconnect and regress, the endothelial cells constituting this previously perfused vessel accomplish to seal it off from the active circulation without causing hemorrhage.

Here, we use time-lapse imaging to analyze the cellular mechanisms that take place during the pruning of a defined blood vessel in the eye of zebrafish embryos. Our analysis reveals that angiogenic sprouting initially leads to the formation of a Yshaped blood vessel branch, which is subsequently resolved. This process entails the rearrangement of endothelial cells within the pruning blood vessel from a multicellular to a partially unicellular tube. We show that blood flow is an important regulator of the pruning event. Importantly, our results suggest that loss of perfusion in itself does not lead to blood vessel pruning, but that pruning might be facilitated by the establishment of differences in blood flow between vessels in a branch point.

\section{Results and Discussion}

\section{Live Imaging of Blood Vessel Pruning}

To better understand vascular remodeling, we set out to identify blood vessels that undergo pruning during zebrafish embryonic development. We performed time-lapse analysis of the eye vasculature between 32 hours post fertilization (hpf) and $48 \mathrm{hpf}$ in $\operatorname{Tg}(k d r l: H s a . H s R A S-m c h e r r y)^{s a 916}$; $\operatorname{Tg}(\text { fli1a:nEGFP })^{y 7}$ fish to label endothelial cell membranes and nuclei (Figure 1). These movies revealed that a discrete portion of the Cranial Division of the Internal Carotid Artery (CrDI) was pruned in a consistent manner (Figure 1, see also Movie S1). Initially, the Nasal Ciliary Artery (NCA) connected to the CrDI, dividing it into a dorsal and a ventral part (Figure 1C, arrow in D, naming according to [11]). After branch point establishment, we observed a collapse of endothelial lumen in the dorsal CrDI (Figure 1E, arrowhead), followed by blood vessel regression (Figure 1E, F, arrowheads). Imaging several zebrafish larvae furthermore showed that this pruning event occurred in a highly reproducible manner, 

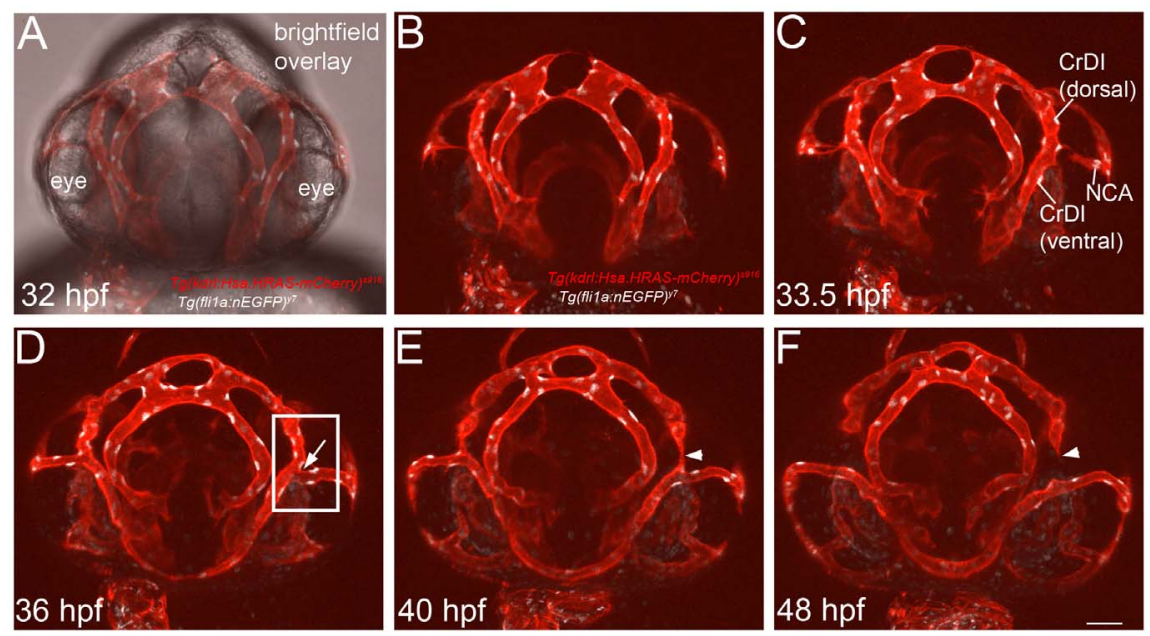

Figure 1. Pruning of the Cranial Division of the Internal Carotid Artery (CrDI) during eye blood vessel development in zebrafish embryos. Still images of a frontal view time-lapse movie of $T g(k d r l: H s a . H R A S-m c h e r r y)^{s 916}$; Tg(fli1a:nEGFP) ${ }^{y 7}$ zebrafish embryos. (A) Overlay of brightfield image with fluorescent channels. (B) Fluorescent channel only. (C) Onset of the connection of the Nasal Ciliary Artery (NCA) to the CrDI at $33.5 \mathrm{hpf}$. (D) Connection of the NCA has completed (arrow). Box indicates area imaged in Figure 2. (E) Collapse of endothelial lumen in the dorsal CrDI (arrowhead) at $40 \mathrm{hpf}$. (F) Completion of CrDI pruning (arrowhead). Scale bar $=50 \mu \mathrm{m}$.

doi:10.1371/journal.pone.0075060.g001

facilitating the investigation of the effects of experimental manipulations on the regression of this blood vessel.

\section{Blood Vessels Rearrange from Multicellular to Partially Unicellular Tubes Prior to Pruning}

To better understand the cellular mechanisms that accompany blood vessel pruning, we generated $\mathrm{Tg}(k d r l: c y \text { tobow 1.0) })^{m u 125}$ fish (see Materials and Methods) to label individual endothelial cells in different colors. Initially, endothelial cells of the dorsal CrDI formed a multicellular tube, with two cells surrounding the vascular lumen (Figure 2A, $34.5 \mathrm{hpf}$ time point, bracket indicates dorsal CrDI with a green and a purple cell, NCA with purple arrow, see also Movie S2). Subsequently, cell rearrangements led to the establishment of a partially unicellular tube in the dorsal $\mathrm{CrDI}$, as evidenced by a single green cell making a connection to the NCA (Figure 2A, 37.5 hpf time point, red bracket). Afterwards, this cell started to disconnect from the NCA, thus causing the separation of the two vessels (Figure 2A, 40 hpf time point). These observations suggest that CrDI pruning occurs via an intermediate step, in which a segment of a multicellular tube changes its architecture into a unicellular tube.

To corroborate our findings, we used $\operatorname{Tg}(U A S$ :VE-cadherin-deltaC$E G F P)^{u b s 12}$ fish [12] to analyze endothelial cell junctional dynamics during CrDI pruning. Initially, all blood vessels within the Yshaped branch consisted of multicellular tubes, as defined by the presence of two or more parallel cell junctions along the vessel axis (Figure 2B, C, $36 \mathrm{hpf}$ to $40.1 \mathrm{hpf}$ time points; see Figure 2D for schematized view, Movie S3). At the onset of dorsal CrDI pruning, one of the two cells at the contact point retracted into the dorsal CrDI, thus transforming the initial multicellular tube into a partially unicellular or seamless tube, as evidenced by the absence of a junction (red brackets in Figures 2B, C, $42.5 \mathrm{hpf}$ time point, Movie S3). Finally, the junctional ring at the attachment site constricted into a single point as the CrDI detached (Figure 2B, C, $45 \mathrm{hpf}$ time point). Thus, as evidenced by our single cell labeling experiments and by the analysis of junctional dynamics, CrDI pruning consists of a multi-step process that involves the rearrangement of endothelial cells from a multicellular into a partially unicellular tube prior to blood vessel detachment and regression.

\section{Macrophage Independent Endothelial Cell Apoptosis Contributes to Blood Vessel Pruning}

Previous studies had shown that macrophage induced apoptosis was essential for the regression of hyaloid vessels during mouse development [5,13-16]. We therefore analyzed if apoptosis was similarly important during CrDI pruning. Observation of endothelial cell nuclei in 9 different time-lapse movies revealed that each regressing dorsal CrDI contained 3-4 endothelial cell nuclei (counted in 9 time lapse movies, Table S1 in File Supplementary Tables). Of these, 1-2 endothelial cells died during CrDI regression (Figure 3A, A', cell undergoing apoptosis is labeled by yellow arrow at time point $44.25 \mathrm{hpf}$, which marks nuclear fragmentation, Movie S4). The remaining CrDI cells migrated towards the dorsally located Primordial Midbrain Channel and in 2 out of 9 movies into the ventral CrDI. Therefore, CrDI pruning is accompanied by endothelial cell death and incorporation of the remaining endothelial cells into neighboring blood vessels.

To address the question of whether the observed apoptosis was required for blood vessel regression, we overexpressed the apoptosis inhibitor $b c l 2$ [17]. This blocked endothelial cell apoptosis (6 out of 6 time-lapse movies; Figure 3B, B') without affecting overall morphology (Figure 3C). In addition, the number of apoptotic cells in all tissues was reduced, as shown by Acridine Orange staining (Figure 3D). Blocking apoptosis led to a pruning defect in $20 \%$ of the embryos analyzed (Figure 3E, Table S2 in File S1). However, we observed that in the $b c l 2$ injected embryos more endothelial cells migrated into the neighboring blood vessels. We therefore conclude that even though endothelial cell apoptosis occurs during CrDI pruning, it is not absolutely required for the completion of this process.

Next, we analyzed if the observed endothelial cell apoptosis is dependent on macrophages. We injected morpholinos (MO) targeting the macrophage specific transcription factor pul.1 [18]. pu.1 MO injected embryos had normal regression in $95.1 \%$ of 352 eyes analyzed, compared to $97.8 \%$ in control embryos (Table $\mathrm{S} 3$ in File $\mathrm{S} 1$ ). These results indicate that 


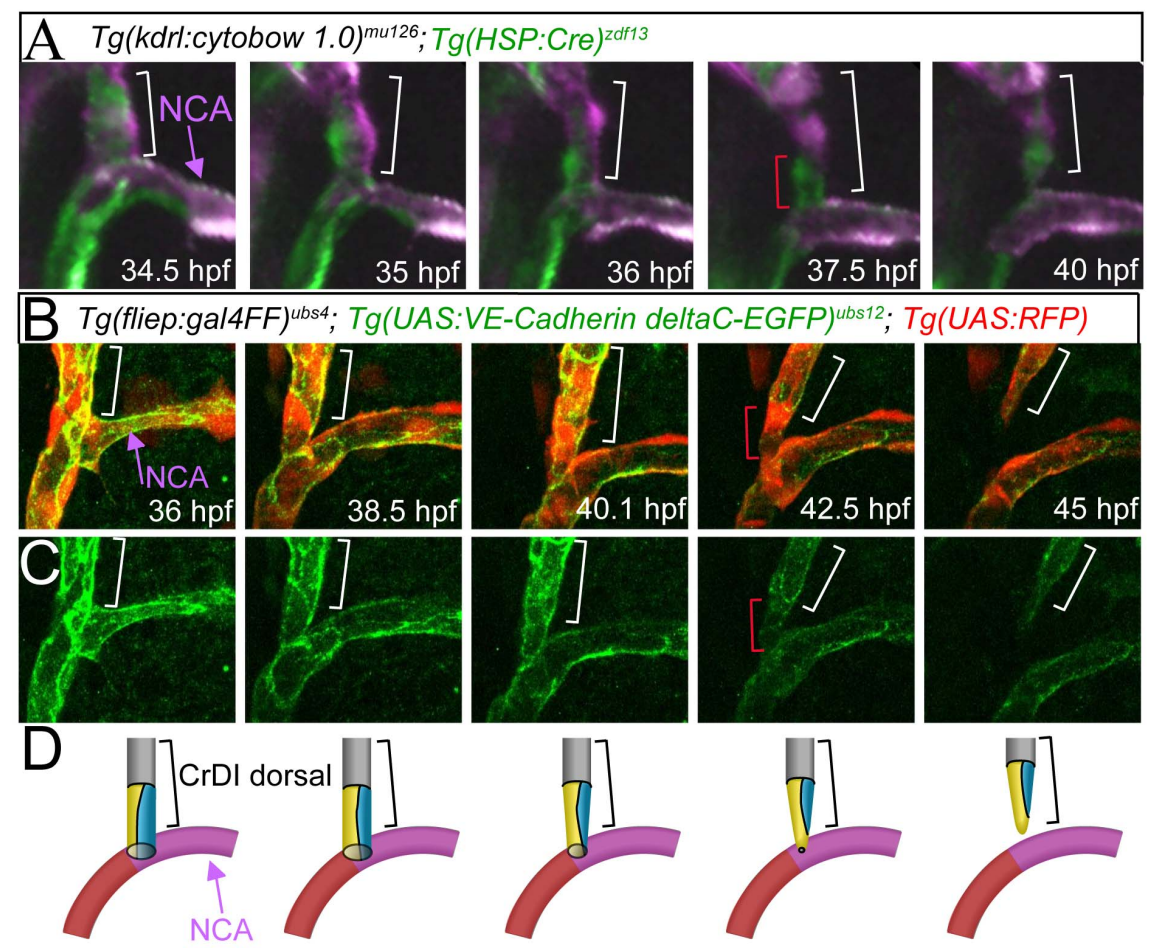

Figure 2. Endothelial cells rearrange from a multicellular into a partially unicellular tube during blood vessel pruning. (A) Still images from a time-lapse movie of $\mathrm{Tg}\left(\mathrm{kdrl}:\right.$ :cytobow 1.0) ${ }^{\mathrm{mu} 125} ; \mathrm{Tg}(\mathrm{HSP}: \mathrm{Cre})^{\text {zdf13 }}$ embryos. Imaged area indicated with box in Figure 1D. Bracket marks dorsal $\mathrm{CrDI}$. At the $37.5 \mathrm{hpf}$ time point, note unicellular connection between the dorsal CrDI and the NCA (single green cell, marked with red bracket). (B) Merged still images from time-lapse imaging of Tg(UAS:RFP); Tg(fliep:Gal4FF) ${ }^{\text {ubs4 }}$; Tg(UAS:VE-cadherin-deltaC-EGFP) $)^{\text {ubs } 12}$ between 36 and 45 hpf. Red bracket at $42.5 \mathrm{hpf}$ indicates unicellular connection between dorsal CrDI and NCA. (C) Still images from Tg(UAS:VE-cadherin-deltaC-EGFP) ${ }^{\text {ubs } 12}$ expression corresponding to images in B. (D) Schematic drawing of cell rearrangements shown in B, C. Brackets label dorsal CrDI, purple arrow highlights NCA.

doi:10.1371/journal.pone.0075060.g002

macrophages do not influence CrDI regression. This suggests that the mechanisms controlling CrDI pruning are distinct from those regulating the regression of the hyaloid vasculature.

\section{Changes in Hemodynamics Regulate Blood Vessel Pruning}

Previous studies analyzing blood vessel pruning in zebrafish brains, mice retinae and airways showed that loss of blood vessel perfusion precedes blood vessel regression [7,10,19,20]. We therefore monitored changes in blood flow patterns between 36 and $48 \mathrm{hpf}$ in CrDI and NCA blood vessels (Figure 4A, Movie S5). Prior to connection of the NCA to the CrDI, no erythrocytes were detected within the sprouting NCA (Figure 4A, 34 hpf time point). Upon connection to the CrDI, red blood cells entered the NCA, while blood flow through the dorsal CrDI became progressively weaker (Figure 4A, $38 \mathrm{hpf}$ ). Eventually, blood flow was almost completely rerouted through the NCA, while the dorsal CrDI was only minimally perfused (Figure 4A, $39 \mathrm{hpf}$ time point). Subsequently, the dorsal CrDI regressed (Figure 4A, 41 and $48 \mathrm{hpf}$, labeled with blue arrow). Therefore, connection of the NCA to the CrDI leads to distinct changes in blood flow within the dorsal CrDI and the NCA, respectively. Furthermore, a reduction of blood flow preceded pruning of the dorsal CrDI.

Based on these observations, we set out to determine whether manipulating blood flow patterns would influence CrDI pruning. We asked, whether we could establish forced blood flow through the dorsal CrDI by laser ablating the NCA. We performed the laser ablation on one side of experimental embryos, while we left the other side as a control. We observed a persistent dorsal CrDI, which continued to carry blood flow (data not shown), on the side on which we had ablated the NCA, but not on the control side (Figure 4B, B', 4C, C', Movie S6). These results suggest that in the absence of an NCA connection, forced blood flow through the dorsal CrDI prevents blood vessel pruning.

We next asked whether inhibition of blood flow would lead to precocious pruning of the CrDI. We manipulated blood flow by altering the heartbeat either by drug treatment or by injecting zebrafish embryos with MO targeting cardiac troponin $t 2 a$ (tnnt $2 a$ ), which never establish a heartbeat [21]. Surprisingly, in tnnt2a MO injected embryos, the CrDI failed to prune in about $50 \%$ of cases (Table S4 in File Supplementary Tables). In embryos that were either treated with $4 \mathrm{x}$ Tricaine or with Nifedipine to stop the heartbeat [22], the CrDI similarly failed to prune in $60 \%$ of cases (Figure 4D, white brackets label persistent CrDI, blue arrow marks regressed CrDI in control embryos, Figure 4E, Table S5 in File Supplementary Tables). Reducing the heartbeat by $20 \%$ through treatment with $3 \mathrm{x}$ Tricaine blocked CrDI regression in around $40 \%$ of the embryos (Figure 4E, Tables S5, S6 in File S1). To test, whether this block in CrDI pruning was reversible, we performed wash out experiments, in which we blocked the heartbeat for 4 hours from 34 to $38 \mathrm{hpf}$ and from 38 to $42 \mathrm{hpf}$. For both treatment regimes, $\mathrm{CrDI}$ regression was blocked during the time without heartbeat, but started after drug wash out and reached almost normal levels at 48 hpf (Figure 4F, Table S7 in File S1). These findings indicate that solely losing perfusion in a given blood vessel is not sufficient to trigger pruning in a situation of global 


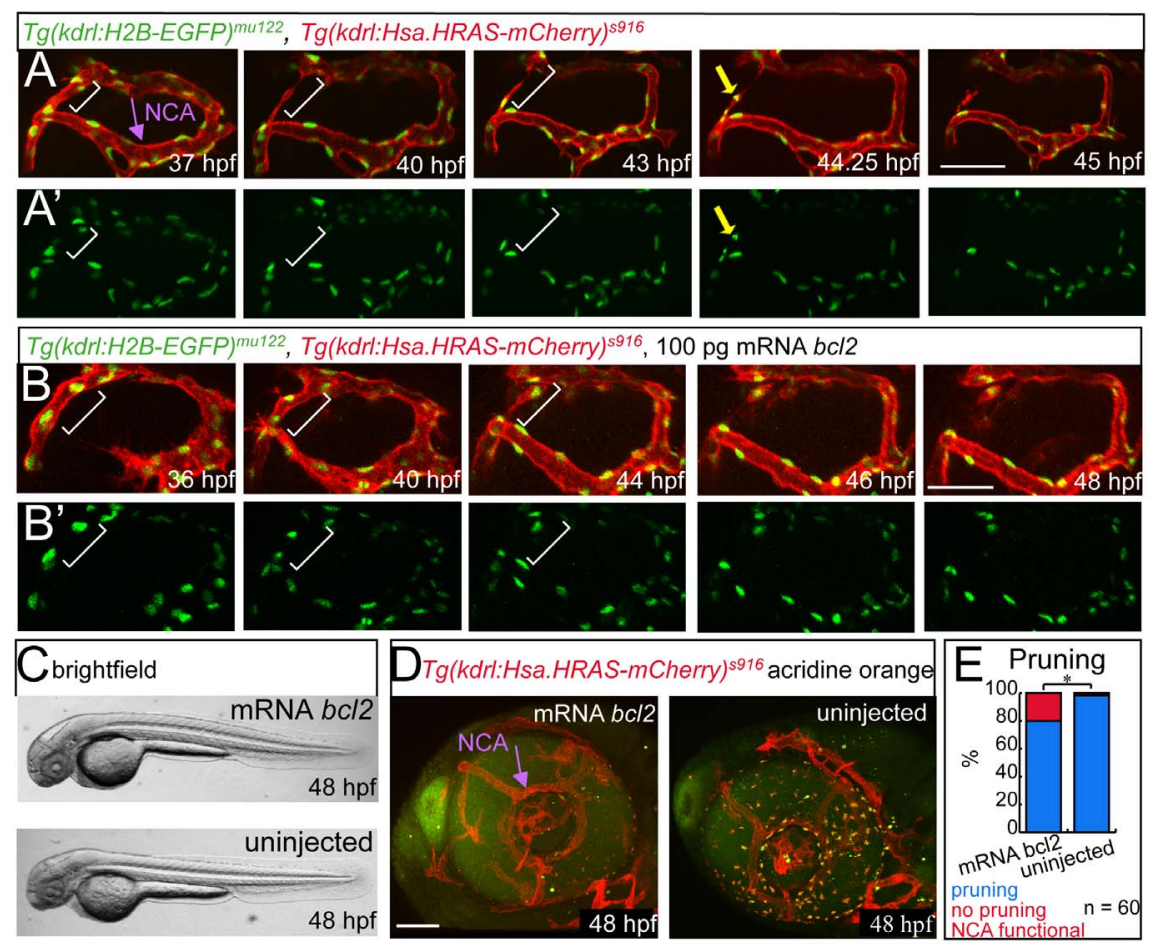

Figure 3. Endothelial cell apoptosis during CrDI pruning. All images in lateral view with anterior to the left. (A) Stills of time-lapse imaging of Tg(kdrl:H2B-EGFP) ${ }^{\text {mu122 }}, \mathrm{Tg}(\mathrm{kdrl}: H s a \text {.HRAS-mCherry) })^{516}$ from 37-45 hpf. (A') Tg(kdrl:H2B-EGFP) ${ }^{\text {mu122 }}$ channel only. White brackets indicate dorsal CrDI. Yellow arrow in (A) and $\left(\mathrm{A}^{\prime}\right)$ at $42.25 \mathrm{hpf}$ time point points to dying cell. (B) Stills of time- lapse imaging of $T g(k d r l: H 2 B-E G F P)^{\text {mu122; }}$ Tg(kdrl:Hsa.HRAS$m$ Cherry) ${ }^{5916}$ embryos from 36-48 hpf injected with bc/2 RNA. No dying cells are detectable in the CrDI. (B') Tg(kdrl:H2B-EGFP) ${ }^{\text {mu122 }}$ channel only. (C) Brightfield images of $b c / 2$ mRNA or uninjected embryos. (D) Acridine orange staining on $b c / 2$ mRNA injected or control embryos. (E) Quantification of CrDl pruning at $48 \mathrm{hpf}$.

doi:10.1371/journal.pone.0075060.g003

block of blood flow, in which adjacent blood vessels would also not be perfused.

Our study is the first to describe the cellular rearrangements occurring during blood vessel pruning and establishes a sequence of events during which a multicellular tube is transformed into a partially unicellular tube prior to blood vessel detachment. This change in blood vessel architecture might be necessary in order to ensure proper blood vessel pruning without vessel rupture or hemorrhage. A recent report describes that during blood vessel fusion, endothelial cells rearrange from a unicellular to a multicellular tube [12]. Therefore, the changes in blood vessel architecture that occur during blood vessel pruning appear to be the same as for blood vessel fusion, but in the reverse order.

Previous studies showed that pruning of early forming hindbrain vessels in zebrafish embryos occurs normally in the absence blood flow [23,24], while at later stages, loss of perfusion leads to accelerated blood vessel pruning [10]. We observed loss of blood flow prior to CrDI pruning, while forcing blood flow through the CrDI led to a persistent CrDI. Of interest, global block of blood flow did not lead to precocious CrDI pruning, but to an impairment of CrDI pruning. These results suggest that it is not loss of blood flow per se that determines if a given blood vessel will prune. Rather, the difference in blood flow between neighboring blood vessels might be sensed by endothelial cells, ultimately triggering the pruning of the less perfused vessel. Our blood flow blocking experiments furthermore suggest that blood flow directly acts on endothelial cells during blood vessel pruning, since pruning rapidly resumed after reestablishing the heartbeat. We note that in embryos with a global block in blood flow, CrDI pruning still occurred in about $40 \%$ of analyzed embryos, suggesting the existence of flow independent mechanisms controlling blood vessel pruning. The identification of these mechanisms and their potential integration with flow-based processes will greatly advance our understanding of blood vessel pruning and vascular remodeling.

\section{Materials and Methods}

\section{Zebrafish Strains}

Zebrafish were maintained as described previously [25]. All animal experiments were performed in accordance with the relevant laws and institutional guidelines at the Max Planck Institute for Molecular Biomedicine. All protocols concerning animal maintenance and handling were approved by the state of North Rhine-Westphalia (Germany), and all efforts were made to minimize suffering. Embryos were staged by hours post-fertilization (hpf) at $28.5^{\circ} \mathrm{C}$ [26]. The following transgenic lines were used:

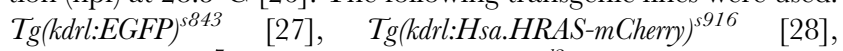

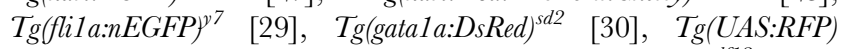
(kindly provided by Kawakami lab), $\operatorname{Tg}(\text { HSP:Cre })^{z d f 13}$ [31]; Tg(UAS:VE-cadherin-deltaC-EGFP) ubs 12 [12], Tg(kdrl:cytobow1.0 $)^{\text {mu126 }}$ and $T g(k d r l: H 2 B-E G F P)^{m u 122}$ (this study).

\section{Generation of $T g(k d r l: H 2 B-E G F P)^{m u 122}$ and Tg(kdrl:cytobow1.0) ${ }^{\text {mu126 }}$ Zebrafish}

pCS2-H2B-EGFP [32] containing Danio rerio Histone2B (H2B) fused in frame to EGFP was used to PCR amplify and subclone H2B-EGFP into pBluescript. Thereafter, H2B-EGFP was cloned via EcoRV and EcoRI into pKdrl:MCS-Tol2 [33] to 


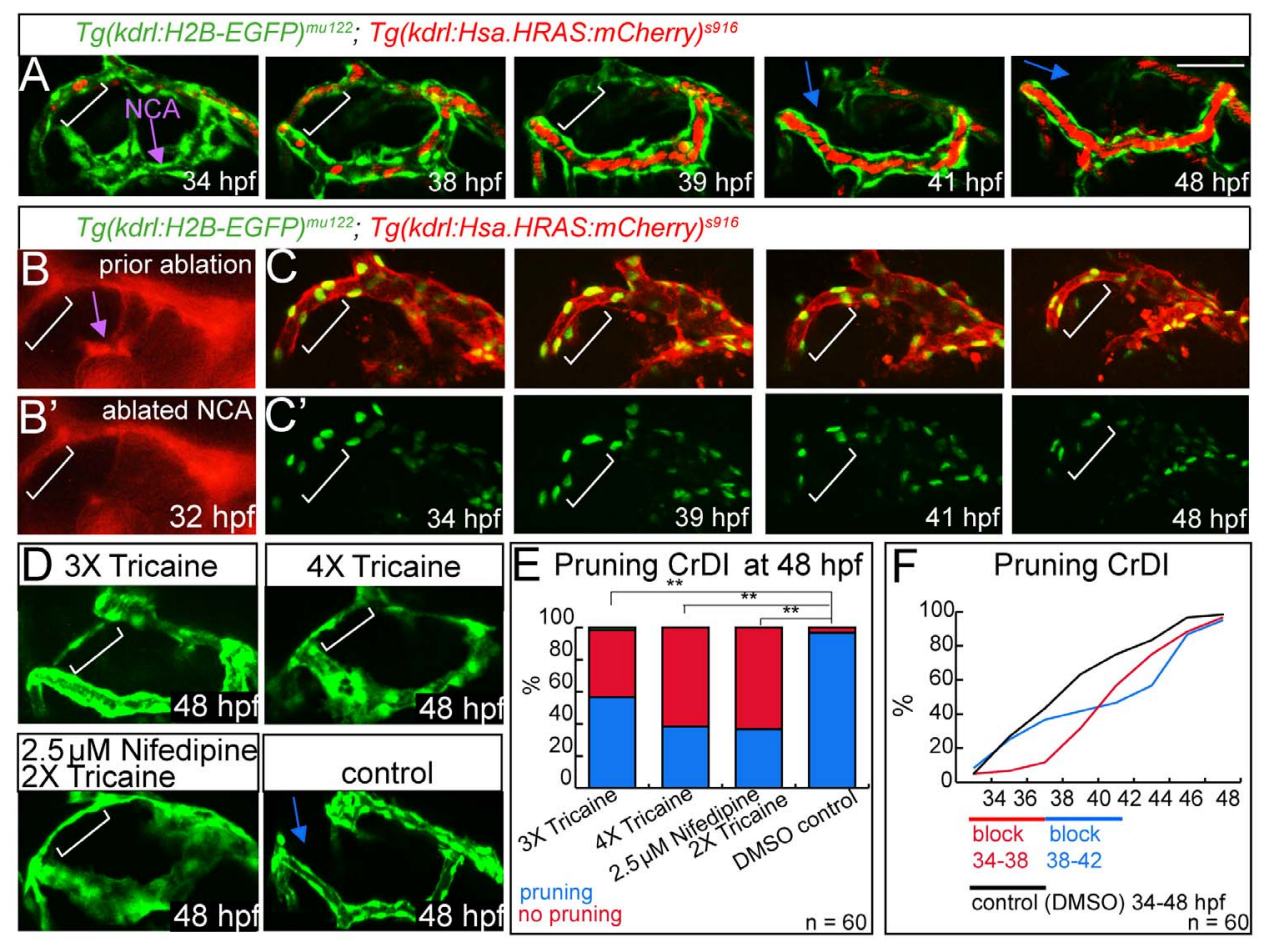

Figure 4. Pruning of the CrDI is flow dependent. Lateral views, anterior to the left, dorsal to the top. (A) Still images of time-lapse of $T g(k d r l: E G F P)^{s 843} ;$ Tg(gata 1a:DsRed) ${ }^{\text {sd2 }}$ from 34-48 hpf. White brackets indicate dorsal CrDl, purple arrow indicates NCA. Note reduction of blood flow in dorsal CrDI (39 hpf time point) prior to regression (blue arrows at 41 and $48 \mathrm{hpf}$ time points). (B) Laser ablation of NCA at $32 \mathrm{hpf}$ (purple arrow),

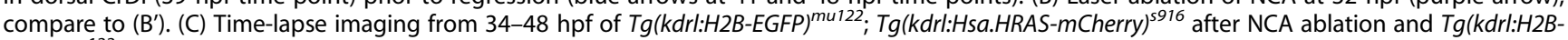
$E G F P)^{m u 122}$ only $\left(C^{\prime}\right)$. Note persistent CrDI (white brackets). (D) Blocking of heartbeat by indicated drug treatments from $30-48 \mathrm{hpf}$ and imaging at $48 \mathrm{hpf}$. (E) Quantification of drug treatments shown in D. (F) Influence of $4 \mathrm{hr}$ block of heartbeat followed by drug wash out on CrDI regression at indicated time points. Scale bar $=50 \mu \mathrm{m}$.

doi:10.1371/journal.pone.0075060.g004

generate pKdrl:MCS-Tol2-H2B-EGFP. DNA was isolated by Maxiprep, purified with PCR purification kit (Qiagen) and $\mathrm{AB}$ embryos at the one cell stage were injected with $30 \mathrm{ng}$ DNA pKdrl:MCS-Tol2-H2B-EGFP and $50 \mathrm{ng}$ mRNA tol2 transposase (Message Machine kit, Ambion) [34]. At 24 hpf, embryos were screened for vascular-specific EGFP expression. Adult founders with germline transmission were identified by outcrosses and the brightest founder was maintained to establish $\mathrm{Tg}(\mathrm{kdrl}: \mathrm{H} 2 \mathrm{~B}$ EGFP) ${ }^{\text {mu122 }}$

In order to generate $T g(k d r l: c y t o b o w 1.0)^{m u 126}$ fish, we used NheI and DraIII to release the cassette containing the fluorescent proteins from CMV Brainbow 1.0 "L" (Addgene 18721) [35]. Subsequently, we filled in the restriction sites with T4 DNA polymerase and ligated the blunted insert into EcoRV digested pkdrl:MCS-Tol2 plasmid [33]. We screened for adult founders with germline transmission by analyzing vascular tdTomato expression at $36 \mathrm{hpf}$ and maintained the brightest founder to establish $\mathrm{Tg}(k d r l \text { :cytobow 1.0 })^{\text {mu126}}$. Cytobow 1.0L constructs contain two floxed fluorescent proteins (tdTtomato and Cerulean) and Yellow Fluorescent Protein. Cre-mediated recombination between individual Lox-P sites can lead to the expression of different combinations of fluorescent proteins, as previously shown [35]. In order to gain temporal control over Cre activity, we crossed Tg(kdrl:cytobow 1.0 $)^{\text {mu125 }}$ fish to $\operatorname{Tg}(\text { HSP:Cre })^{z d f 13}$ fish. This resulted in $25 \%$ double transgenic offspring. Embryos were subsequently heat shocked at $90 \%$ epiboly and those with differently labeled endothelial cells in anterior blood vessels were chosen for timelapse analysis.

\section{Analysis of Regression of CrDI Artery and Statistics}

We used $T g(k d r l: E G F P)^{s 843}$ embryos to analyze the regression of the dorsal CrDI. Regression was analyzed at $48 \mathrm{hpf}$ under a stereomicroscope or via confocal microscopy. Only embryos with normal blood flow patterns at $36 \mathrm{hpf}$ were included in the analysis. We defined regression as complete loss of endothelial connection between $\mathrm{CrDI}$ and PMBC. Each experiment was performed in triplicates with 20 eyes being analyzed per experiment. Quantification charts display the average values of three experiments. Data were evaluated with two-tailed unpaired Student's t test and significance was calculated for regression.

\section{Time-lapse, Confocal Microscopy, and Vessel Ablation}

Live imaging of endothelial cell junctions using the $\operatorname{Tg}(U A S: V E-$ cadherin-deltaC-EGFP) ${ }^{u b s 12}$ line was performed as previously described [12]. For other in vivo imaging, live embryos were mounted essentially as described previously [36]. Briefly, embryos were mounted in $1 \%$ low melting point agarose in E3 embryo medium with $168 \mathrm{mg} / 1$ Tricaine (1X) for anesthetization and $0.003 \%$ phenylthiourea to inhibit pigmentation. Imaging was carried out at an SP5 confocal microscope using a 20x objective (Leica Microsystems). We used a heated microscope chamber at $28.5^{\circ} \mathrm{C}$ for recording time- lapse movies. Stacks were taken every $15 \mathrm{~min}$ with a step size of $1.5-2.5 \mu \mathrm{m}$. Confocal stacks and time-lapse movies were analyzed using Imaris Software (Bitplane), Image J (NIH), and Quicktime Player Pro. Vessels in close proximity to the CrDI were cropped away to increase clarity. Ablation of the NCA artery was carried out between $31-33 \mathrm{hpf}$ using a Zeiss epifluorescence microscope equipped with a PALM micro laser 
dissection system. The laser beam was applied until fluorescence of $T g(k d r l: H 2 B-E G F P)^{m u 122}$ disappeared in the ablated vessel. After NCA ablation, we monitored the embryos for PMBC integrity and normal blood flow.

\section{Morpholinos (MOs) and mRNA Injection}

$\mathrm{MO}$ were obtained from Gene-Tools and dissolved in $\mathrm{dH}_{2} \mathrm{O}$. Embryos were injected at one cell stage with $4 \mathrm{ng} \mathrm{MO}$ tnnt $2 a$ (silent heart 5' CATGTTTGCTCTGATCTGACACGCA 3') [21] or $4 \mathrm{ng}$ MO pu.1 (5'-GATATACTGATACTCCATTGGTGGT-3') [18]. As a control, we injected standard control morpholinos (5'-CGTCTTACGTCAGTTACAATTTATA-3'). Danio rerio bcl2 mRNA was transcribed from pCS2Bcl2 (kindly provided by J. Hillmer) using Message Machine kit (Ambion) and injected into one cell stage with a final amount of $100 \mathrm{pg}$.

\section{Acridine Orange (AO) Staining and Drug Treatments}

For AO staining, embryos were incubated for $30 \mathrm{~min}$ in $10 \mu \mathrm{g} /$ $\mathrm{ml} \mathrm{AO}$ in E3 at $28.5^{\circ} \mathrm{C}$, followed by washing for $30 \mathrm{~min}$. Drug treatments were carried out on dechorionated embryos in E3 medium. To completely stop the heart at various time points, we either treated zebrafish embryos with $4 \mathrm{X}$ Tricaine or with $2 \mathrm{X}$ Tricaine in addition to $2.5 \mu \mathrm{M}$ Nifedipine [22]. To slow down heartbeat, we treated embryos with $3 \mathrm{X}$ Tricaine. All drug treatments were performed from 30 to $48 \mathrm{hpf}$, prior to the onset of $\mathrm{CrDI}$ regression. For wash out experiments, drugs were removed and embryos were rinsed twice with E3 medium. We observed that after drug wash out, blood flow was re-established within 1 hour.

\section{Supporting Information}

\section{File S1 Supporting tables.}

(DOG)

Movie S1 (Related to Figure 1): Time lapse imaging of early eye blood vessel development. Time-lapse imaging of Tg(kdrl:Hsa.HRAS-mCherry $)^{s 916}$; Tg(fli1a:nEGFP $)^{y 7}$ embryos. Images were taken from $32-48 \mathrm{hpf}$ at 15 minute time intervals. Endothelial cell membranes are marked by red fluorescence, while endothelial cell nuclei are marked in white. Arrows indicate the forming Nasal Ciliary Artery (NCA). After connection of the NCA (indicated by arrows) to the Cranial Division of the internal carotid artery (CrDI), the dorsal CrDI prunes (arrowheads).

(MOV)

Movie S2 Gellular rearrangements during GrDI pruning based on labeling of individual endothelial cells

\section{References}

1. Adams RH, Alitalo K (2007) Molecular regulation of angiogenesis and lymphangiogenesis. Nat Rev Mol Cell Biol 8: 464-478.

2. Geudens I, Gerhardt H (2011) Coordinating cell behaviour during blood vessel formation. Development 138: 4569-4583.

3. Potente M, Gerhardt H, Carmeliet P (2011) Basic and therapeutic aspects of angiogenesis. Cell 146: 873-887.

4. Taniguchi H, Kitaoka T, Gong H, Amemiya T (1999) Apoptosis of the hyaloid artery in the rat eye. Ann Anat 181: 555-560.

5. Lobov IB, Rao S, Carroll TJ, Vallance JE, Ito M, et al. (2005) WNT7b mediates macrophage-induced programmed cell death in patterning of the vasculature. Nature 437: 417-421.

6. Diez-Roux G, Lang RA (1997) Macrophages induce apoptosis in normal cells in vivo. Development 124: 3633-3638.

7. Lobov IB, Cheung E, Wudali R, Cao J, Halasz G, et al. (2011) The Dll4/Notch pathway controls postangiogenic blood vessel remodeling and regression by modulating vasoconstriction and blood flow. Blood 117: 6728-6737.
(Related to Figure 2A). Time-lapse imaging of $T g(k d r l: c y t o b o w$ 1.0) ${ }^{\text {mu126 }} ; \mathrm{Tg}(\mathrm{HSP}: \mathrm{Cr})^{z d f 13}$ embryos. Images were taken from 34.5$40 \mathrm{hpf}$ at 15 minute time intervals. Distinct endothelial cells were false colour labeled in green and magenta, respectively. Note absence of magenta cell in dorsal CrDI during unicellular configuration prior to $\mathrm{CrDI}$ pruning.

(MOV)

Movie S3 Gellular rearrangements during GrDI pruning based on labeling of endothelial cell junctions (Related to Figure 2B, G). Time-lapse imaging with Tg(UAS:RFP); Tg(fliep:Gal4FF) ${ }^{u b s}$; $T g(U A S: V E$-cadherin-deltaC-EGF$P)^{u b s 12}$ in a frontal-lateral orientation between 36 and $45 \mathrm{hpf}$. In the overlay channel, endothelial cells are labeled in red and endothelial junctions are shown in green. Middle panels show junctional labeling alone with individual cells and junctions colour coded to illustrate junctional dynamics during CrDI pruning. Right panel shows RFP channel alone, marking endothelial cell bodies.

(MOV)

\section{Movie S4}

(MOV)

\section{Movie S5}

(MOV)

Movie S6 Laser ablation of the NGA and influence on CrDI pruning (Related to Figure 4B). Laser ablation of the NCA was carried out at $32 \mathrm{hpf}$ and time-lapse imaging was performed from $34-48 \mathrm{hpf}$ in $T g(k d r l: H 2 B-E G F P)^{m u 122}$, Tg(kdrl:Hsa.HRAS-mCherry $)^{s 916}$ double transgenic zebrafish, labeling all endothelial nuclei in green and endothelial membranes in red. In the absence of a functional NCA, the CrDI fails to prune. (MOV)

\section{Acknowledgments}

We would like to thank Janine Hillmer for providing zebrafish $b c l 2$ and Wiebke Herzog for providing the $\operatorname{Tg}(H S P: C r e)^{z d f 13}$ fish. We would also like to thank Reinhild Bussmann for excellent fish care and Inna Herzog for technical assistance. We are thankful to Wade Sugden for critically reading of the manuscript. We would furthermore like to thank the imaging facility of the University of Basel.

\section{Author Contributions}

Conceived and designed the experiments: EK MA HGB AFS. Performed the experiments: EK AL AFS. Analyzed the data: EK MA HGB AL AFS. Contributed reagents/materials/analysis tools: EE LH. Wrote the paper: EK AFS.
8. Phng LK, Potente M, Leslie JD, Babbage J, Nyqvist D, et al. (2009) Nrarp coordinates endothelial Notch and Wnt signaling to control vessel density in angiogenesis. Dev Cell 16: 70-82.

9. Yashiro K, Shiratori H, Hamada H (2007) Haemodynamics determined by a genetic programme govern asymmetric development of the aortic arch. Nature 450: $285-288$.

10. Chen O, Jiang L, Li C, Hu D, Bu JW, et al. (2012) Haemodynamics-driven developmental pruning of brain vasculature in zebrafish. PLoS Biol 10: e1001374.

11. Isogai S, Horiguchi M, Weinstein BM (2001) The vascular anatomy of the developing zebrafish: an atlas of embryonic and early larval development. Dev Biol 230: 278-301.

12. Lenard A, Ellertsdottir E, Herwig L, Krudewig A, Sauteur L, et al. (2013) In Vivo analysis reveals a highly stereotypic morphogenetic pathway of vascular anastomosis. Dev Cell 25: 492-506.

13. Lang R, Lustig M, Francois F, Sellinger M, Plesken H (1994) Apoptosis during macrophage-dependent ocular tissue remodelling. Development 120: 33953403. 
14. Lang RA, Bishop JM (1993) Macrophages are required for cell death and tissue remodeling in the developing mouse eye. Cell 74: 453-462.

15. Meeson A, Palmer M, Calfon M, Lang R (1996) A relationship between apoptosis and flow during programmed capillary regression is revealed by vital analysis. Development 122: 3929-3938.

16. Meeson AP, Argilla M, Ko K, Witte L, Lang RA (1999) VEGF deprivationinduced apoptosis is a component of programmed capillary regression. Development 126: 1407-1415.

17. Liu TX, Howlett NG, Deng M, Langenau DM, Hsu K, et al. (2003) Knockdown of zebrafish Fancd2 causes developmental abnormalities via p53-dependent apoptosis. Dev Cell 5: 903-914.

18. Rhodes J, Hagen A, Hsu K, Deng M, Liu TX, et al. (2005) Interplay of pu.1 and gatal determines myelo-erythroid progenitor cell fate in zebrafish. Dev Cell 8: 97-108.

19. Baffert F, Le T, Sennino B, Thurston G, Kuo CJ, et al. (2006) Cellular changes in normal blood capillaries undergoing regression after inhibition of VEGF signaling. Am J Physiol Heart Circ Physiol 290: H547-559.

20. Baluk P, Lee CG, Link H, Ator E, Haskell A, et al. (2004) Regulated angiogenesis and vascular regression in mice overexpressing vascular endothelial growth factor in airways. Am J Pathol 165: 1071-1085.

21. Sehnert AJ, Huq A, Weinstein BM, Walker C, Fishman M, et al. (2002) Cardiac troponin $\mathrm{T}$ is essential in sarcomere assembly and cardiac contractility. Nat Genet 31: 106-110.

22. Bussmann J, Wolfe SA, Siekmann AF (2011) Arterial-venous network formation during brain vascularization involves hemodynamic regulation of chemokine signaling. Development 138: 1717-1726.

23. Corti P, Young S, Chen CY, Patrick MJ, Rochon ER, et al. (2011) Interaction between alk1 and blood flow in the development of arteriovenous malformations. Development 138: 1573-1582.

24. Fujita M, Cha YR, Pham VN, Sakurai A, Roman BL, et al. (2011) Assembly and patterning of the vascular network of the vertebrate hindbrain. Development 138: $1705-1715$
25. Westerfield M (1993) The Zebrafish Book. Eugene, OR: University of Oregon Press.

26. Kimmel CB, Ballard WW, Kimmel SR, Ullmann B, Schilling TF (1995) Stages of embryonic development of the zebrafish. Dev Dyn 203: 253-310.

27. Jinn SW, Beisl D, Mitchell T, Chen JN, Stainier DYR (2005) Cellular and molecular analyses of vascular tube and lumen formation in zebrafish. Development 132: 5199-5209.

28. Hogan BM, Bos FL, Bussmann J, Witte M, Chi NC, et al. (2009) ccbel is required for embryonic lymphangiogenesis and venous sprouting. Nature Genetics 41: 396-398.

29. Roman BL, Pham VN, Lawson ND, Kulik M, Childs S, et al. (2002) Disruption of acvrll increases endothelial cell number in zebrafish cranial vessels. Development 129: 3009-3019.

30. Traver D, Paw BH, Poss KD, Penberthy WT, Lin S, et al. (2003) Transplantation and in vivo imaging of multilineage engraftment in zebrafish bloodless mutants. Nature Immunology 4: 1238-1246.

31. Le X, Langenau DM, Keefe MD, Kutok JL, Neuberg DS, et al. (2007) Hea shock-inducible Cre/Lox approaches to induce diverse types of tumors and hyperplasia in transgenic zebrafish. Proc Natl Acad Sci U S A 104: 9410-9415.

32. Zanella C, Campana M, Rizzi B, Melani C, Sanguinetti G, et al. (2010) Cells segmentation from 3-D confocal images of early zebrafish embryogenesis. IEEE Trans Image Process 19: 770-781.

33. Blum Y, Belting HG, Ellertsdottir E, Herwig L, Luders F, et al. (2008) Complex cell rearrangements during intersegmental vessel sprouting and vessel fusion in the zebrafish embryo. Dev Biol 316: 312-322.

34. Kawakami K, Takeda H, Kawakami N, Kobayashi M, Matsuda N, et al. (2004) A transposon-mediated gene trap approach identifies developmentally regulated genes in zebrafish. Dev Cell 7: 133-144.

35. Livet J, Weissman TA, Kang H, Draft RW, Lu J, et al. (2007) Transgenic strategies for combinatorial expression of fluorescent proteins in the nervous system. Nature 450: 56-62.

36. Lawson ND, Weinstein BM (2002) In vivo imaging of embryonic vascular development using transgenic zebrafish. Dev Biol 248: 307-318. 